\title{
A COMPARISON OF SMART PHONE CAMERA CALIBRATION APPROACHES USING THE DIGITAL CLOSE-RANGE PHOTOGRAMMETRY TECHNIQUE
}

\author{
Hossam El-Din Fawzy \\ Associate Professor, Civil Engineering Department, Faculty of Engineering, \\ Kafr El-Sheikh University, Kafr El-Sheikh, Egypt.
}

\begin{abstract}
This paper presents a real-time application of mobile phone digital camera calibration adopting two different methods depends on Photo Modeler computer software to determine the distortion parameters and the camera intrinsic. Two different methods were used to assess the calibration process via with two different mathematical models, which are the DLT and the SIFT, to detect and identify the features of the calibration pattern to assess a robust bundle orientation of the targeted geometrical shapes. The results showed that both methods have a standard difference of estimation of the pattern calibration values that do not exceed 1.70 pixels in both $X$, $Y$ direction with a maximum connectivity of minimum $94 \%$ between images, in counter of the Auto-Calibration approach which has a residuals of 2.60 pixels in both direction of $X$ and, $Y$ with a connectivity of $35 \%$ as a maximum overlapping percentage between images.
\end{abstract}

Keywords: Mobile Phone Camera, Camera Calibration, Photo Modeler, Accuracy and Close-Range Photogrammetry

Cite this Article: Hossam El-Din Fawzy, A Comparison of Smart Phone Camera Calibration Approaches Using the Digital Close-Range Photogrammetry Technique, International Journal of Civil Engineering and Technology, 11(10), 2020, pp. 40-48. https://iaeme.com/Home/issue/IJCIET?Volume $=11 \&$ Issue $=10$

\section{INTRODUCTION}

Nowadays, computer vision and digital analysis are developing to be one of the most important fields of modern technology and applications, which presents robust approaches at the digital close-range photogrammetry (DCRP) application such as, real-time data collection and manipulation to achieve the best results in short time period. The DCRP technique became applicable though the digital cameras, especially the dedicated cell-phone camera as a reliable tool for evaluating the distortion error of the camera lens to be adjusted for many geometrical applications such as architectural photogrammetry, civil engineering, surveying 
photogrammetry, and many industrial photogrammetry [1] in addition to the crime scene analyzation and multimedia [2]. During recent years, the manufacturers and developers of mobile phones enhanced the possibilities of digital technology to offer the opportunity of the image capturing professionally in the abundance of illumination [3].

This research presents the application of several approaches regrading the calibration of the digital phone camera that comes with NOKIA Lumia 640xl, to determine the interior parameters of the camera intrinsic coefficient such as the focal length $(f)$, camera sensor format size $(F x, F y)$ and the distortion factors $\mathrm{k}_{1}, \mathrm{k}_{2}, \mathrm{k}_{3}, \mathrm{P}_{1}$ and $\mathrm{P}_{2}$ [4]. To estimate these values, a robust bundle mathematical model should be applied technically to achieve the multi view of the orientation. Direct Linear Transform (DLT) presents the ability to estimate the distortion parameters [5] as a computer application software Photo Modeler adopted this software in which it proved a real accurate photogrammetric measurement technique $[6,7,8]$. A comparison of the camera calibration results are assessed within several calibration techniques in a methodology of,

- Plan Pattern method.

- Self-calibration technology.

\section{METHODOLOGY}

\subsection{Plan Pattern Method}

Plane pattern method depends on creating a standard pattern contains basic shapes of circles, Fig.1, or standard squares which are known as "chess board" template, Fig.2. This method was proposed by Zhang and $\mathrm{Li}$ [9] as a technique of the multi orientation images capturing to create a homography. It is linear relationship between the estimated coordinates of the template images and the coordinates of the stations of imagery (camera position). To solve such linear relation all coordinates are tabled as a matrix explains the relations that control the imaging process including the distortion parameters, these parameters are manipulated by the DLT technique as following [5];

$$
\left.\begin{array}{l}
f=x+\Delta x-\frac{C_{1} X+C_{2} Y+C_{3} Z+C_{4}}{C_{9} X+C_{10} Y+C_{11} Z+1} \\
g=y+\Delta y-\frac{C_{5} \mathrm{X}+\mathrm{C}_{6} \mathrm{Y}+\mathrm{C}_{7} \mathrm{Z}+\mathrm{C}_{8}}{\mathrm{C}_{9} \mathrm{X}+\mathrm{C}_{10} \mathrm{Y}+\mathrm{C}_{11} \mathrm{Z}+1}
\end{array}\right\}
$$

Where:

$\mathrm{C}_{1}, \mathrm{C}_{2}, \mathrm{C}_{3} \ldots \mathrm{C}_{11}$ are the transformation parameters $\mathrm{X}, \mathrm{V}$ and $\mathrm{Z}$ are the space coordinates of the targeted shapes at the pattern. Equation (1) results from the equation of the central perspective, $\Delta \mathrm{x}, \Delta \mathrm{y}$ are systematic deformations due to the lens distortion of the image, i.e. deviations from the central perspective. Equation (1) can be solved directly for the eleven transformation parameters $\left(\mathrm{C}_{1}: \mathrm{C}_{11}\right)$. So there are, at least, six points in the image that have known object-space coordinates. Equation (1) can be rewritten, to serve in a least squares formulation relating known control points to image coordinate measurements, as;

$$
\left.\begin{array}{c}
V_{u}=C_{1} X+C_{2} Y+C_{3} Z+C_{4}-x C_{9} X-x C_{10} Y-x C_{11} Z-\Delta x \\
V_{v}=C_{5} X+C_{6} Y+C_{7} Z+C_{8}-y X-y C_{10} Y-y C_{11} Z-\Delta y
\end{array}\right\}
$$

Estimating of the lens distortion: 


$$
\left.\begin{array}{l}
\Delta x=x^{-}\left(d_{1} s^{2}+d_{2} s^{4}+d_{3} s^{6}\right)+e_{1}\left(s^{2}+2 x^{-2}\right)+2 e_{2} x^{-} y^{-} \\
\Delta y=y^{-}\left(d_{1} s^{2}+d_{2} s^{4}+d_{3} s^{6}\right)+2 e_{1} x^{-} y^{-}+2 e_{2}\left(s^{2}+2 y^{-}\right)
\end{array}\right\}
$$

Where:

$$
x^{-}=x-x o \quad y^{-}=y-y o \quad s^{2}=(x-x o)^{2}+(y-y o)^{2}
$$

$x, y$ are the image coordinates. $e_{1}, e_{2}$ are two asymmetric parameters for decentring distortion. $d_{1}, d_{2}$ and $d_{3}$ are a radial distortion of the three symmetric parameters. $\mathrm{s}$ is the radial distance of the principal point $x o, y o$.

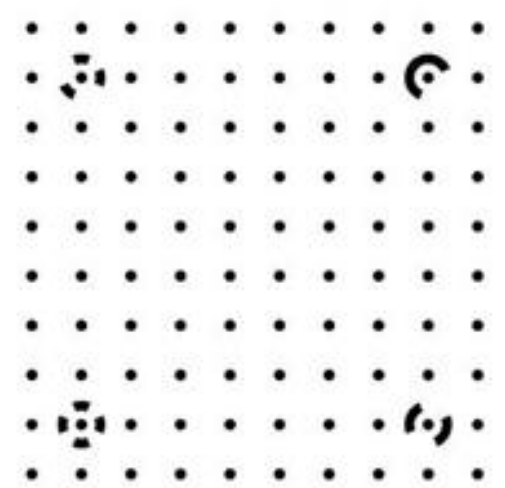

Figure 1 Photo Modeler Calibration Pattern

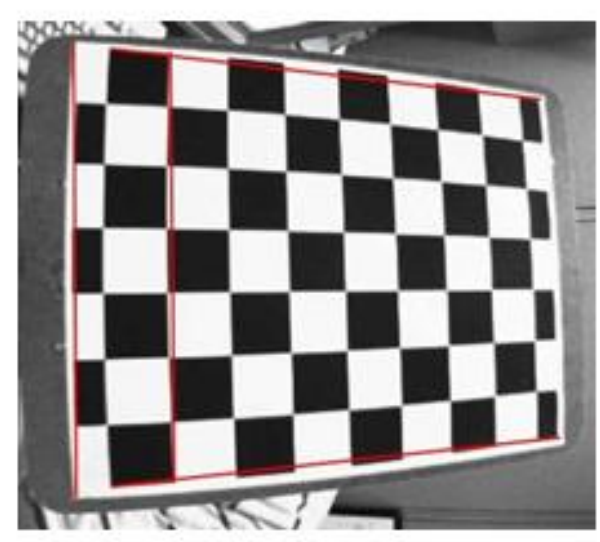

Figure 2 Chess calibration board template

\section{SELF-CALIBRATION TECHNOLOGY}

Self-calibration technology method needs a high technology to adjust the scene and the features obtained to match with different views such as the visual reality or gaming construction. So, it does not depend on the specific geometrical shapes as the first method. Moreover, it has a noticed lack of robustness and durability [10]. The feature detection plays an important role in scene detection, identification, the matching and orientation process. Through the current work SIFT algorithm was adopted to detect the corners and blobs of the scene using the same standard pattern for just assessing the comparison between both methods within the same captured images.

\subsection{Digital Camera Calibration Procedures}

By using a single calibration pattern Fig. 1 was printed on an A4 sheet that contains 4 control points, the left-down corner will be considered as a principle point (coordinates of $0,0,0$ ). Fixing the A4 sheet on a flat surface that all points included in the sheet have a $\mathrm{Z}$ coordinate $=$ 0 and the other points $\mathrm{X}, \mathrm{Y}$ values differenced in constant value determined by the software. Three digital images were captured in $(0,90,180)$ degree respectively around the orientation point of the camera lens of each side of the sheet by a handle operator. A set of total captured pictures equal 12 (which is enough number of images to determine the lens unknown distortion values $\left(d_{1}, d_{2}, d_{3}, e_{1}, e_{2}\right.$, and the camera format size of both methods [7]).

The used camera has a specification of 13MP sensor and all processing operations are conducted by a computer with a processor of Core i5 $6200 \mathrm{u}, 6.30 \mathrm{GHz}$ and $12 \mathrm{~GB}$ of RAM, internal storage $1 \mathrm{~Tb}$ hard disk 
A Comparison of Smart Phone Camera Calibration Approaches Using the Digital Close-Range Photogrammetry Technique

\section{RESULT AND DISCUSSION}

\subsection{DLT Calibration Results}

Calibration results report after images analyzing shows the values of camera parameters, the time that needed to conduct that method does not exceed 4 minutes with a fast and accurate determination of the camera parameters, accompanied with an estimation of the deviation of images in pixel, to be easy to apply the camera and the calibration project idealization as shown in Table 1. Figures 3 and 4 show the trending of the quality of the points after image idealization, the maximum error does not exceed 2.00 pixel and a mean value of 0.6 pixel of the localization of the points which assess a distortion estimation of 32 pixel at the middle of each image in $\mathrm{Y}$ direction and 48 pixel in $\mathrm{X}$ direction. The photogrammetric project assesses connectivity and overlapping reaches $99.0 \%$, Fig.5. All the calibration images were accepted and used during the calibration process, Fig.6.

\begin{tabular}{|c|c|c|c|c|c|}
\hline 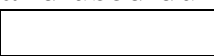 & Table 1 I & igital camera & Lumia $640 \mathrm{XI}$ & calibration re & esults \\
\hline Image No. & $\alpha$ in degrees & $\beta$ in degrees & $\kappa$ in degrees & \begin{tabular}{|c|} 
Max. and \\
overall \\
residual error
\end{tabular} & $\begin{array}{c}\text { Max. image distortion } \\
\text { in pixels after camera } \\
\text { idealization }\end{array}$ \\
\hline 1 & -0.704108 & -39.040722 & -90.686895 & & \\
\hline 2 & 0.893891 & -30.164367 & -179.451150 & & \\
\hline 3 & -0.721902 & -34.154241 & 89.516247 & & \\
\hline 4 & 35.427787 & 0.381468 & 179.944729 & & \\
\hline 5 & 28.254773 & -0.486689 & -89.773458 & & \\
\hline 6 & 38.669121 & -0.129342 & -0.010968 & MRE: 1.5301 & $Y$ direction $=32$ \\
\hline 7 & -2.150142 & 24.713161 & -0.200913 & ORE: $0.3079<$ & $X$ direction $=48$ \\
\hline 8 & -37.376075 & 0.151546 & -0.090490 & 2.00 accepted & \\
\hline 9 & -28.474363 & 1.476261 & 89.674770 & & \\
\hline 10 & -28.874793 & 1.775298 & 89.976728 & & \\
\hline 11 & -36.485251 & 0.681980 & -179.391317 & & \\
\hline 12 & -36.410252 & 0.869841 & 179.609472 & & \\
\hline Camera & libration & & lue & & Deviation \\
\hline \begin{tabular}{|r} 
para \\
\end{tabular} & leters & & & & \\
\hline Focal length $f$ & & 3.5794 & $17 \mathrm{~mm}$ & & $5.4 \mathrm{e}-04 \mathrm{~mm}$ \\
\hline $\mathrm{Xp}$ - image pr & ciple point & 2.2645 & $16 \mathrm{~mm}$ & & $3.5 \mathrm{e}-04 \mathrm{~mm}$ \\
\hline Co.X & & 1.2851 & $45 \mathrm{~mm}$ & & $3.0 \mathrm{e}-04 \mathrm{~mm}$ \\
\hline Yp - image pr & cipal point & 4.5405 & $93 \mathrm{~mm}$ & & $1.6 \mathrm{e}-04 \mathrm{~mm}$ \\
\hline Co.Y & & 2.5525 & $78 \mathrm{~mm}$ & & $0.00 \mathrm{~mm}$ \\
\hline $\mathrm{Fx}$ - format $\mathrm{w}$ & & -1.04 & He-02 & & $5.6 e-05$ \\
\hline Fy - format he & & 1.61 & $e-03$ & & $1.0 \mathrm{e}-05$ \\
\hline$d_{1}-$ radial dis & rtion 1 & & & & 0.00 \\
\hline$d_{2}-$ radial dis & rtion 2 & -7.52 & $5 e-05$ & & $1.1 \mathrm{e}-05$ \\
\hline$d$ - radial disto & ion 3 & -1.46 & Be-04 & & $9.7 e-06$ \\
\hline$e_{1}-$ tangential & listortion 1 & & & & \\
\hline$e_{2}$ - tangential & listortion 2 & & & & \\
\hline
\end{tabular}




\section{Point Quality}

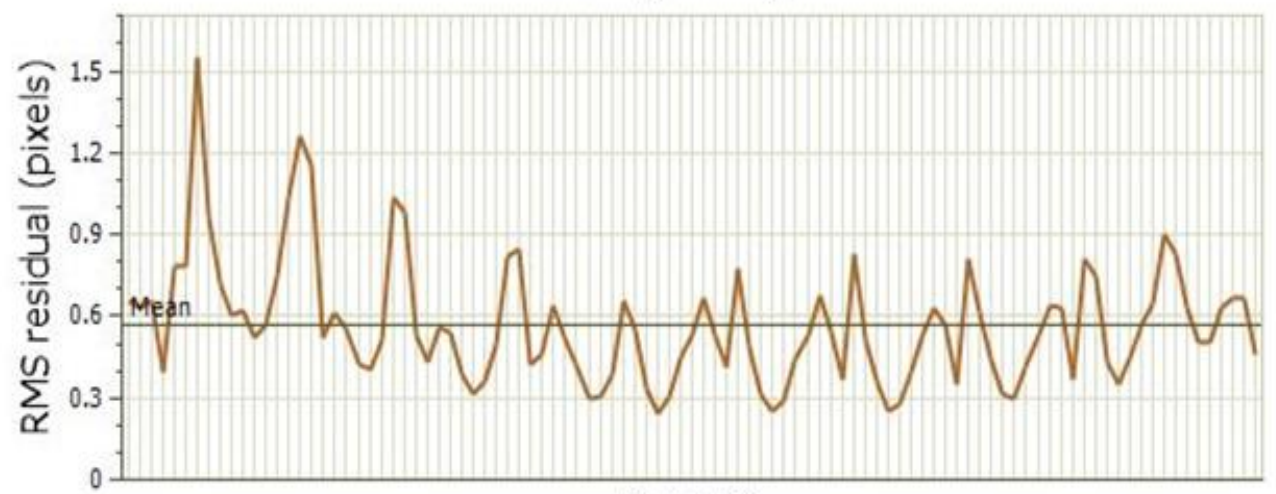

Point ID

Figure 3 Residual error in $\mathrm{X}$ direction

Point Quality

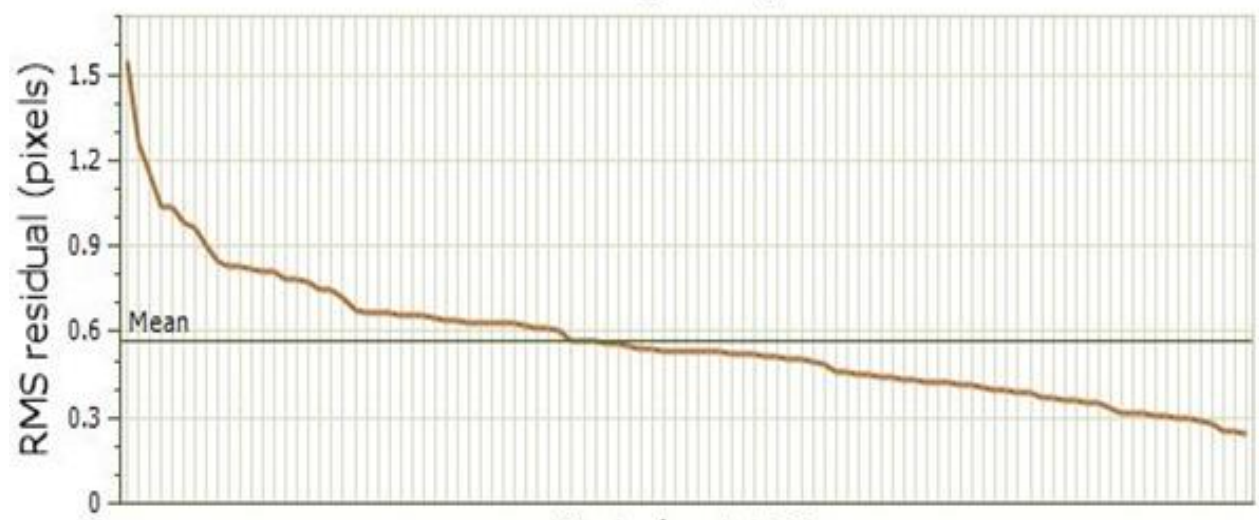

Sorted point ID

Figure 4 Maximum residual error in $\mathrm{Y}$ direction

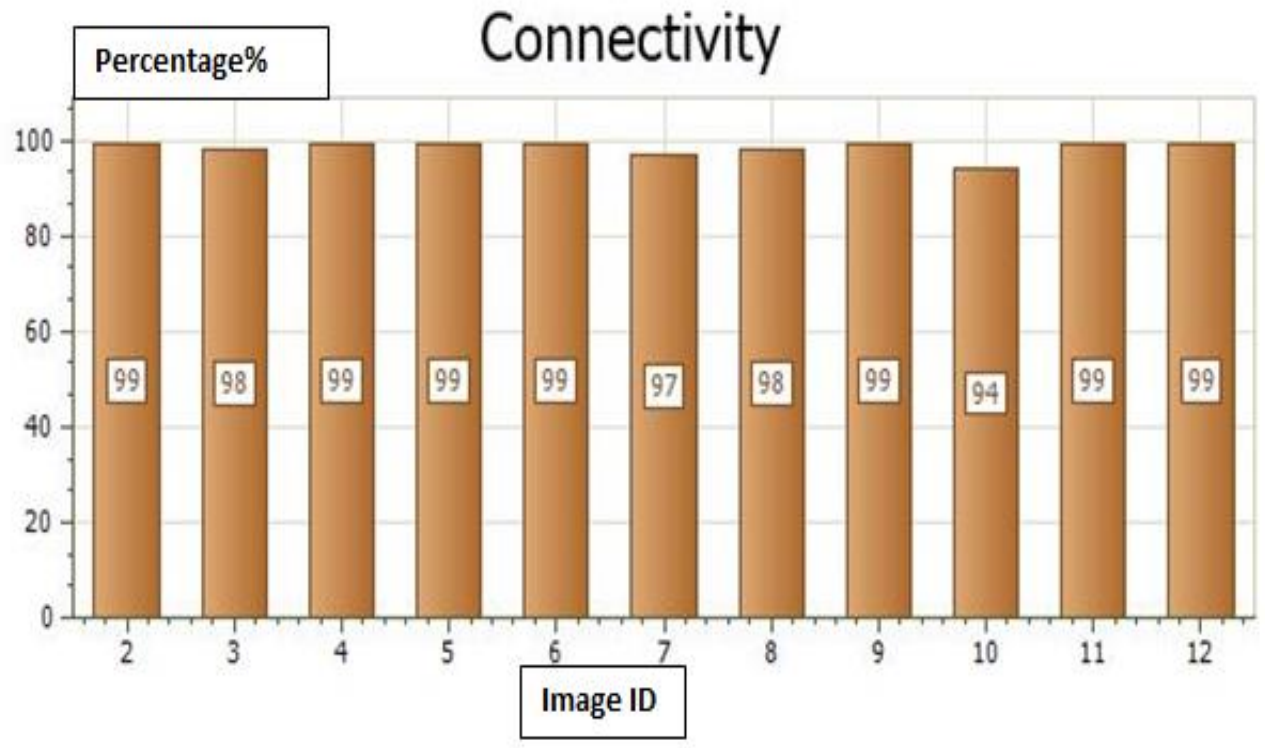

Figure 5 The percentage of the connectivity between images 
A Comparison of Smart Phone Camera Calibration Approaches Using the Digital Close-Range Photogrammetry Technique

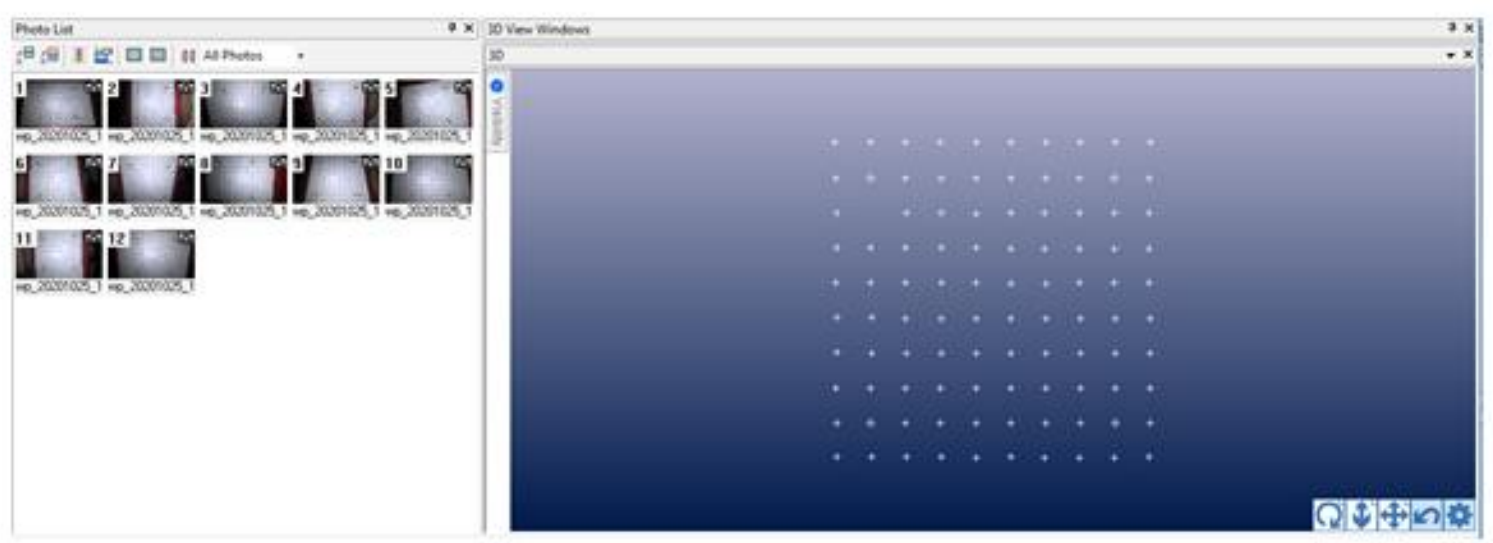

Figure 6 Twelve calibration images were accepted and used at the calibration process

\subsection{Self-Calibration Technology Results}

The same 12 digital images were used to create the self-auto calibration process with the same condition of positioning and illumination. The calibration results show a value of $d_{3}$ which means determining more distortion at the lens of the camera, Table 2.

Results depending on this technology on the feature extraction of the scene, are affected by the lack of illumination Fig.7. The software was not able to create sufficient detection of the overlapping between images, consequently, some of these images were ignored during the feature extraction process which effects directly on the maximum final residual of the images as shown in Figs. $8 \&$ 9. The maximum overall residuals exceeded the 2.00 pixel with very low connectivity of $35.0 \%$ as a maximum value between images were shown in Fig. 10.

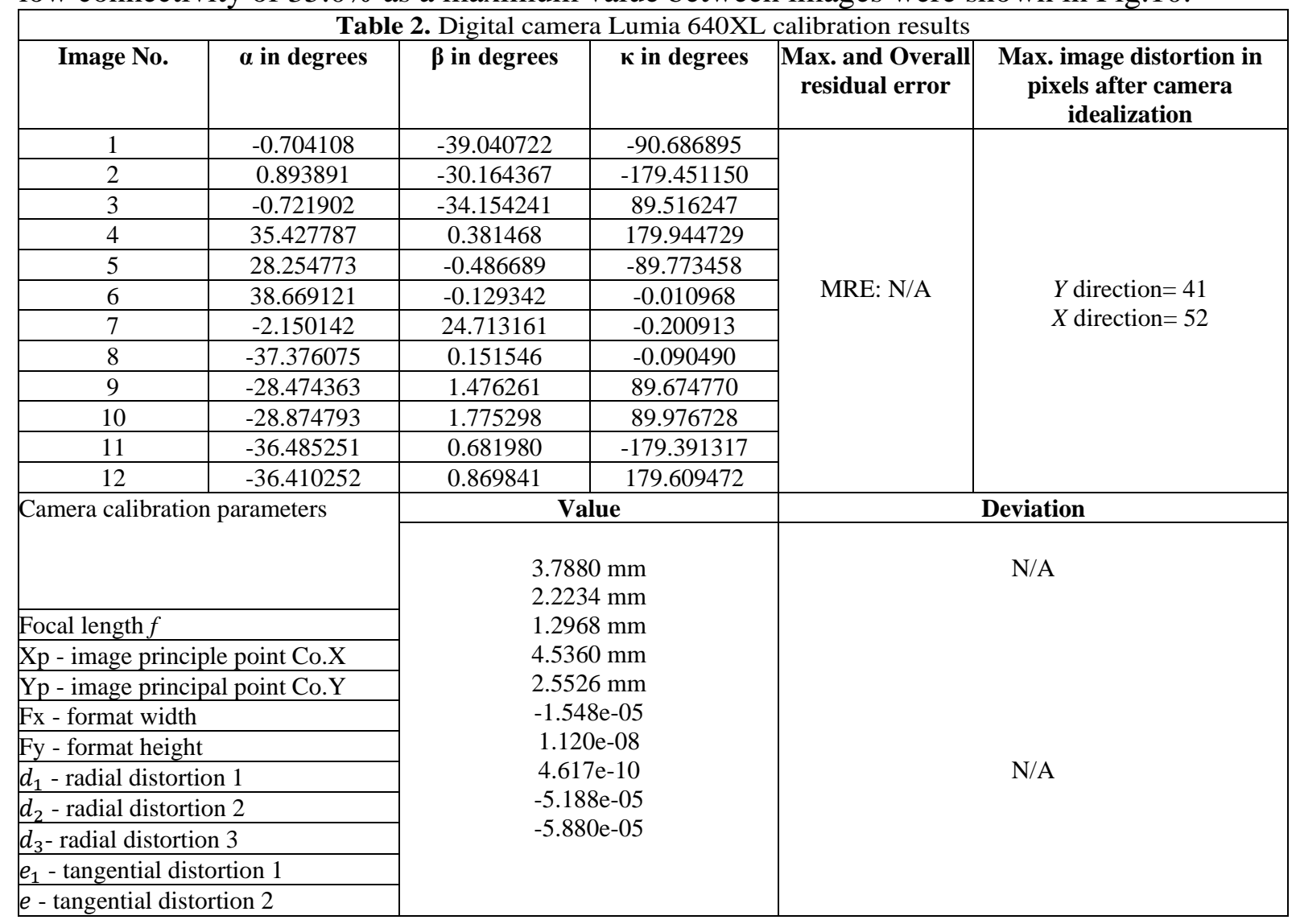




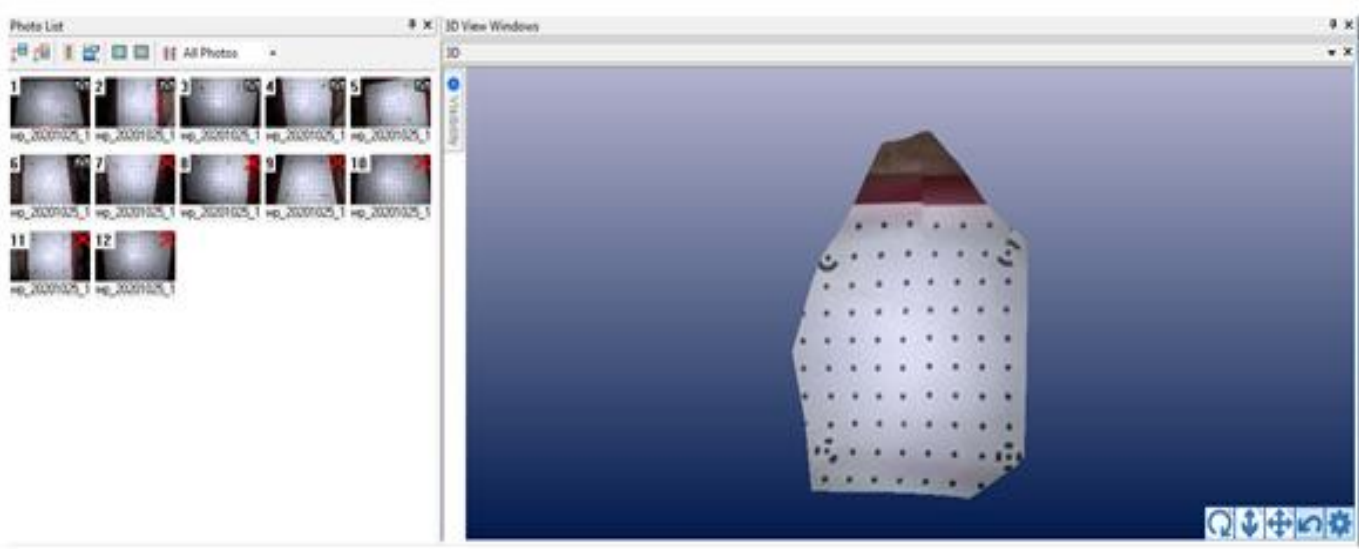

Figure 7 Multi of images were out of the processing due to the insufficient illumination

\section{Point Quality}

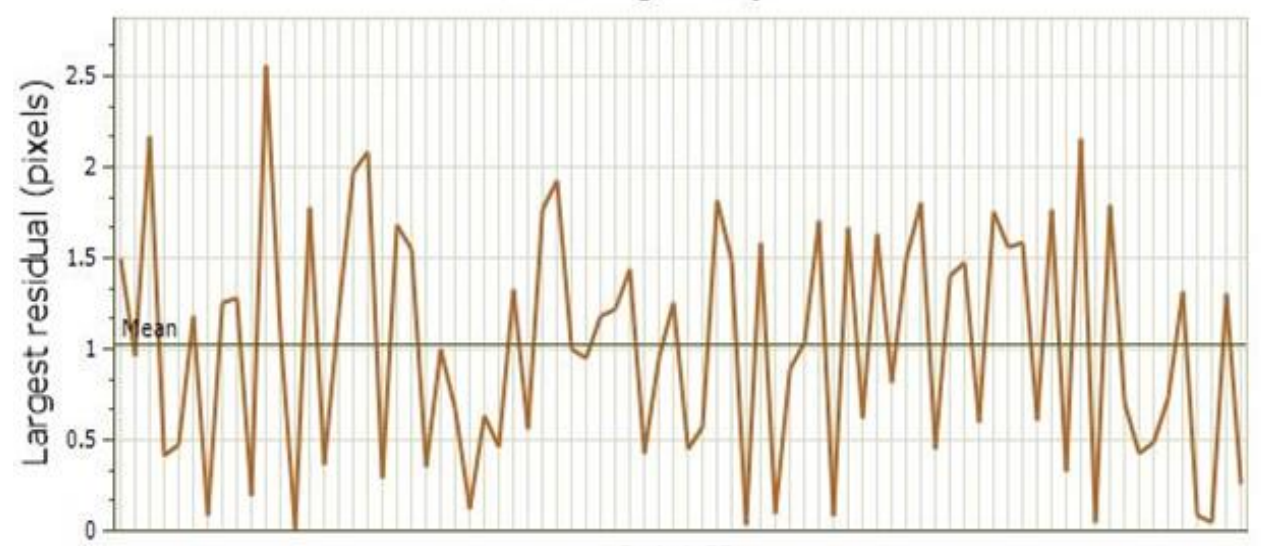

Point ID

Fig.8 Max. Residual error in X direction

Figure 8 Max. Residual error in X direction

\section{Point Quality}

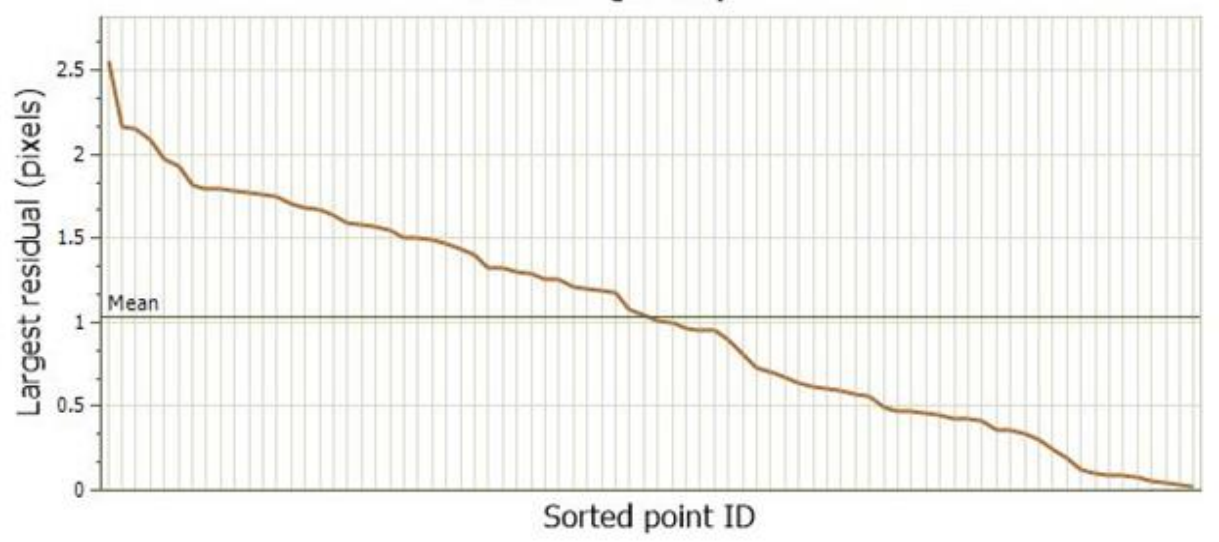

Figure 9 Max. Residual error in Y direction 


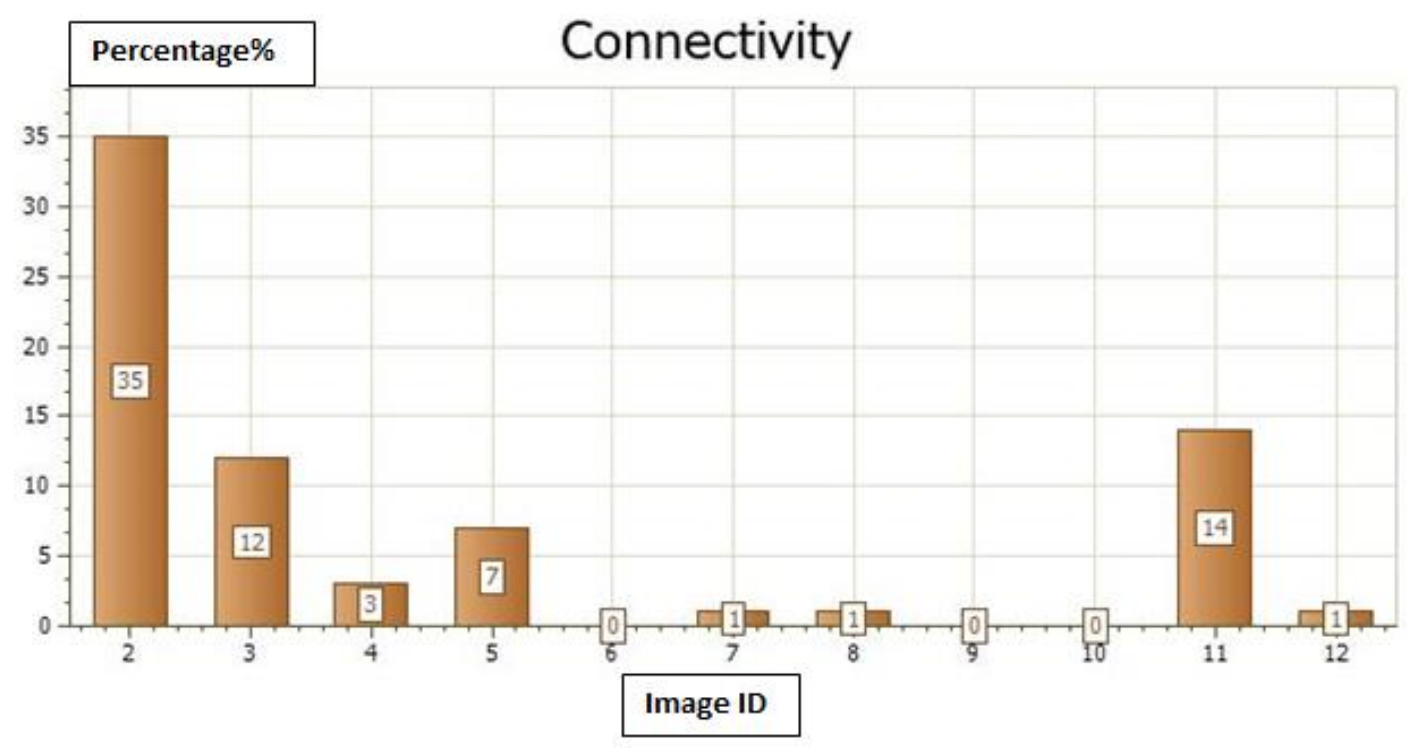

Figure 10 The percentage of the connectivity between images

\section{CONCLUSION}

The DLT method shows robust and fast detection of the calibration pattern without any missing points at the template. The coordinates axis was applied typically on the model to create a quick point coordinates identification with maximum residuals that did not exceed 2.00 pixels in $\mathrm{X}, \mathrm{Y}$ direction with a mean of error 0.60 pixels due to the sufficient overlapping between 12 digital images which assess a percentage of $94.0 \%$ and just two factors of barrel radial distortion $k_{1}, k_{2}$. Alternatively, the Auto-calibration method has more difficulties regarding features detection due to the lack of illumination which caused more residuals of the image's orientation projection and also the final connectivity was less than the DLT method which reaches less than $40.0 \%$. However, the same properties of the imagery were constant within the same covering angles, also, the distortion factors increased at the second approach with a result of $k_{3}$ which means the first approach of pattern calibration is better than the Auto-Calibration method if compared at the constrains.

\section{REFERENCES}

[1] Rogers, M., (2003). "Highway Engineering”, Blackwell, Oxford.

[2] Luhmann, S., Robson, S., Kyle, I., Harley (2011) "Close Range Photogrammetry Principles, techniques and applications". Whittles Publishing.

[3] A. Agapiou, Prof. A. Georgopoulos, 2006. "Photogrammetric Potential of Digital Cameras in Handheld Gadgets for Digital Close Range Applications" The 7th International Symposium on Virtual Reality, Archaeology and Cultural Heritage.

[4] Jason P. de Villiers a, b, F. Wilhelm Leuschnerb et al, (2008) Centi-pixel accurate realtime inverse distortion correction. International Symposium on Opt mechatronic Technologies, California, United States.

[5] Abdel-Aziz, Y. I., 1975 “Asymmetrical Lens Distortion” Civil Engineering studies, Cairo University, Cairo, Egypt, Proceedings of the Photogrammetric engineering \& remote sensing (pp. 337-340).

[6] Eos Systems, Inc., PhotoModeler UAS User's Manual, Vancouverm B.C., Canada, 2018. 


\section{Hossam El-Din Fawzy}

[7] H. El Fawzy "Study the accuracy of digital close range photogrammetry technique software as a measuring tool" Alexandria Eng. J., 58 (2019), pp. 171-179.

[8] Hossam El-Din Fawzy, Ali M. Basha, Marco N. Botross. "Estimating a mathematical formula of soil erosion under the effect of rainfall simulation by digital close range photogrammetry technique" Alexandria Engineering Journal, 2020.

[9] Zhang Guang-jun, Li Xiu-zhi. Foot-eye calibration for mobile robots [J]. Robot, Vol.29, No.3, 230-233, 2007.

[10] Qi, Wang \& Li, Fu \& Zhenzhong, Liu. (2010). Review on Camera Calibration. 3354 - 3358. 10.1109/CCjDC.2010.5498574. 